\title{
AN ALGORITHM FOR MULTIPARAMETRIC MIN MAX 0-1-INTEGER PROGRAMMING PROBLEMS RELATIVE TO THE OBJECTIVE FUNCTION
}

\author{
José Luis Quintero $^{1}$ And Alejandro Crema ${ }^{2}$
}

\begin{abstract}
The multiparametric min max 0-1-Integer Programming (0-1-IP) problem relative to the objective function is a family of min max 0-1-IP problems which are related by having identical constraint matrix and right-hand-side vector. In this paper we present an algorithm to perform a complete multiparametric analysis relative to the objective function.
\end{abstract}

Keywords. 0-1-Integer Programming, multiparametric programming, Bottleneck problem.

\section{INTRODUCTION}

The need for multiparametric analysis in mathematical programming arises from the uncertainty in the data. Recently Greenberg [7] published an annotated bibliography for post-solution analysis including parametric Integer Linear Programming (ILP) problems. Greenberg's bibliography can be searched on the World Wide Web (WWW) [8]. Another bibliography available on the WWW is due to Arsham [1]. Jenkins [9-12] has presented a very simple approach to solve parametric ILP problems based on Geoffrion and Nauss [6]. His methods work by solving an appropriate sequence of non-parametric problems and joining the solutions to complete the parametrical analysis.

Received September 8, 2004. Accepted November 24, 2005.

${ }^{1}$ Departamento de Matemáticas Aplicadas, Facultad de Ingeniería, Universidad Central de Venezuela; quintero jl@tutopia.com

2 Escuela de Computación, Facultad de Ciencias, Universidad Central de Venezuela. Apartado 47002, Caracas 1041-A, Venezuela; acrema@kuaimare.ciens.ucv.ve

(c) EDP Sciences 2006 
Recently we have used the Jenkins's approach in order to design algorithms to solve multiparametric 0-1-ILP problems relative to the right-hand-side vector (Crema [2]), the objective function (Crema [3]) and the constraint matrix (Crema [4]).

A theoretical and algorithmic study for parametric 0-1-ILP problems relative to the objective function, including complexity results, have been written by Thiongane, Nagih and Plateau [17].

In this paper we present an approach, that can be viewed as a generalization of [3], to solve min max multiparametric 0-1-Integer Programming (0-1-IP) problems relative to the objective function.

To the best of our knowledge there are no other algorithms to be applied in the min max case. Our algorithm may be implemented by using any software capable of solving Mixed Integer Linear Programming (MILP) problems.

In Section 2 we study the theory that allow us to design the algorithm to be presented in the same section. Computational experience is presented in Section 3. A summary and further extensions are given in Section 4.

\section{TheORETICAL RESUlts AND The ALGORITHM}

Let $L \in \mathbb{R}^{p}, U \in \mathbb{R}^{p}$ with $L \leq U$, let $D \in \mathbb{R}^{q \times p}, d \in \mathbb{R}^{q}$ and let $\Omega=\left\{\theta \in \mathbb{R}^{p}\right.$ : $L \leq \theta \leq U, D \theta \leq d\}$, let $b \in \mathbb{R}^{m}, A \in \mathbb{R}^{m \times n}$ and let $X=\{x: A x \leq b, \quad x \in$ $\left.\{0,1\}^{n}\right\}$.

Let us suppose that $\Omega \neq \emptyset$ and $X \neq \emptyset$.

The multiparametric min max 0 -1-IP problem relative to the objective function is a family of 0 -1-IP problems which are related by having identical constraint matrix and right-hand-side vector. A member of the family is defined as

$$
(P(\theta)) \quad \min \phi(\theta, x) \quad \text { s.t. } \quad x \in X
$$

where $\phi$ is a continuous function on $\Omega$ for all $x \in X, \theta$ is the vector of parameters, $\theta \in \Omega \subseteq \mathbb{R}^{p}, x$ is a vector of $0 / 1$ variables, and $X$ is the set of feasible solutions which does not depend on the vector $\theta$.

We use the following standard notation: if $T$ is an optimization problem then $F(T)$ denotes its set of feasible solutions and $v(T)$ denotes its optimal value (if it exists).

Note that since $X$ is a finite set then there exits an optimal solution for $P(\theta)$ for all $\theta \in \Omega$.

In the min sum case we have $p=n$ and $\phi(\theta, x)=\theta^{t} x$. The min sum case was studied in a previous work (see Crema [3]). In the min max case we have $p=n$ and $\phi(\theta, x)=\max \left\{\theta_{1} x_{1}, \cdots, \theta_{n} x_{n}\right\}$. The min max case is presented in this paper.

We say that $x^{(1)}, \cdots, x^{(r)}$ is a multiparametrical solution if: $x^{(i)} \in X$ for all $i=1, \cdots, r$ and $\min \left\{\phi\left(\theta, x^{(1)}\right), \cdots, \phi\left(\theta, x^{(r)}\right)\right\}=v(P(\theta))$ for all $\theta \in \Omega$.

Suppose that $x^{(i)} \in X$ for all $i=1, \cdots, r$. Let $g^{(r)}(\theta)=\min \left\{\phi\left(\theta, x^{(1)}\right)\right.$, $\left.\cdots, \phi\left(\theta, x^{(r)}\right)\right\}$. Note that $g^{(r)}(\theta) \geq v(P(\theta))$ for all $\theta \in \Omega$ and if $x^{(r+1)} \in X$ then $g^{(r+1)}(\theta) \leq g^{(r)}(\theta)$ for all $\theta \in \Omega$. 
Let $Q^{(r)}$ be a problem in $(\theta, x)$ defined as:

$$
\left(Q^{(r)}\right) \max g^{(r)}(\theta)-\phi(\theta, x) \text { s.t. } \theta \in \Omega, \quad x \in X \text {. }
$$

Observe that $\theta$ is a vector of decision variables in $Q^{(r)}$.

Note that with $Q^{(r)}$ we are looking for the maximal difference between $v(P(\theta)$ ) and an upper bound function defined by $g^{(r)}(\theta)$. If the maximal difference is zero then we have found $v(P(\theta))$ for all $\theta \in \Omega$ and the analysis was completed, otherwise our algorithm finds $x^{(r+1)}$ and $\theta^{*}$ such that $g^{(r+1)}(\theta) \leq g^{(r)}(\theta)$ for all $\theta \in \Omega$ and $g^{(r+1)}\left(\theta^{*}\right)<g^{(r)}\left(\theta^{*}\right)$.

\section{Lemma 2.1.}

(i) There exists an optimal solution for $Q^{(r)}$.

(ii) $v\left(Q^{(r)}\right) \geq 0$.

(iii) If $v\left(Q^{(r)}\right)=0$ then $v(P(\theta))=g^{(r)}(\theta)$ for all $\theta \in \Omega$.

(iv) If $\left(\theta^{*}, x^{(r+1)}\right)$ is an optimal solution for $Q^{(r)}$ then $x^{(r+1)}$ is an optimal solution for $P\left(\theta^{*}\right)$.

(v) Let $\left(\theta^{*}, x^{(r+1)}\right)$ be an optimal solution for $Q^{(r)}$. If $v\left(Q^{(r)}\right)>0$ then $x^{(i)}$ is not an optimal solution for $P\left(\theta^{*}\right)$ for all $i=1, \cdots, r$ and $g^{(r+1)}\left(\theta^{*}\right)<$ $g^{(r)}\left(\theta^{*}\right)$.

Proof.

(i) Since $\phi(\theta, x)$ is a continuous function on $\Omega$ for all $x \in X$ then $g^{(r)}(\theta)$ is a continuous function on $\Omega$. Therefore, since $X$ is a finite set, $Q^{(r)}$ may be viewed as a finite set of problems with a continuous objective function and a compact set of feasible solutions and then there exits an optimal solution.

(ii) Let $\theta \in \Omega$ and $s$ be an index such that: $g^{(r)}(\theta)=\min \left\{\phi\left(\theta, x^{(1)}\right), \cdots, \phi\left(\theta, x^{(r)}\right)\right\}=\phi\left(\theta, x^{(s)}\right)$.

We have that $\left(\theta, x^{(s)}\right) \in F\left(Q^{(r)}\right)$ and $v\left(Q^{(r)}\right) \geq g^{(r)}(\theta)-\phi\left(\theta, x^{(s)}\right)=0$.

(iii) Let $\theta \in \Omega$. Let $x$ be an optimal solution of $P(\theta)$. We have that $g^{(r)}(\theta)-$ $\phi(\theta, x) \leq v\left(Q^{(r)}\right)=0$ and then $\phi(\theta, x)=v(P(\theta)) \leq g^{(r)}(\theta) \leq \phi(\theta, x)$. Therefore $v(P(\theta))=g^{(r)}(\theta)$.

(iv) Let $x \in X$. If $\phi\left(\theta^{*}, x\right)<\phi\left(\theta^{*}, x^{(r+1)}\right)$ then $g^{(r)}\left(\theta^{*}\right)-\phi\left(\theta^{*}, x\right)>g^{(r)}\left(\theta^{*}\right)-$ $\phi\left(\theta^{*}, x^{(r+1)}\right)=v\left(Q^{(r)}\right)$ and we have a contradiction.

(v) Since $0<v\left(Q^{(r)}\right)=g^{(r)}\left(\theta^{*}\right)-\phi\left(\theta^{*}, x^{(r+1)}\right)$ it follows that $\phi\left(\theta^{*}, x^{(r+1)}\right)<$ $g^{(r)}\left(\theta^{*}\right)$. From (iv) we have that $v\left(P\left(\theta^{*}\right)\right)=\phi\left(\theta^{*}, x^{(r+1)}\right)$ and then: $v\left(P\left(\theta^{*}\right)\right)=\phi\left(\theta^{*}, x^{(r+1)}\right)<g^{(r)}\left(\theta^{*}\right)=\min \left\{\phi\left(\theta^{*}, x^{(1)}\right), \cdots, \phi\left(\theta^{*}, x^{(r)}\right)\right\}$ and $x^{(i)}$ is not an optimal solution for $P\left(\theta^{*}\right)$ for all $i=1, \cdots, r$. Also, $g^{(r+1)}\left(\theta^{*}\right)=\phi\left(\theta^{*}, x^{(r+1)}\right)<g^{(r)}\left(\theta^{*}\right)$.

Since $X$ is a finite set, Lemma 1 proves that the next algorithm provide us a complete multiparametrical analysis. 


\section{The multiparametric algorithm}

Step-0: Find $\theta^{(1)} \in \Omega$. Solve $P\left(\theta^{(1)}\right)$. Let $x^{(1)}$ be an optimal solution.

Step-1: $r=1$.

Step-2: Solve $Q^{(r)}$ and let $\left(\theta^{(r+1)}, x^{(r+1)}\right)$ be an optimal solution.

Step-3: If $v\left(Q^{(r)}\right)=0$ STOP (with $v(P(\theta))=g^{(r)}(\theta)$ for all $\theta \in \Omega$ ).

Step-4: $r=r+1$ and return to step-2.

In order to use the algorithm based on Lemma 1 we need algorithms to solve $Q^{(r)}$ and $P(\theta)$.

In the min sum case $P(\theta)$ is a $0-1$-ILP problem and $Q^{(r)}$ may be rewritten as a 0-1-MILP problem (see Crema [3]).

In the min max case $P(\theta)$ is a bottleneck problem that may be solved by using known specialized algorithms. Also, $P(\theta)$ may be rewritten as a 0-1-MILP problem. $Q^{(r)}$ may be rewritten as a 0-1-MILP problem by using techniques, based on Oral and Kettani [16], as you can see below.

Let $Q L^{(r)}$ be a $0-1$-MILP problem in:

$$
\left(\theta, x, y, z, \delta^{(1)}, \cdots, \delta^{(r)}, w^{(1)}, \cdots, w^{(r)}\right)
$$

defined as:

$$
\begin{gathered}
\left(Q L^{(r)}\right) \quad \max y-z \text { s.t. } \\
\theta \in \Omega, \quad x \in X \\
z \geq L_{i} x_{i} \quad(i=1, \cdots, n) \\
z \geq \theta_{i}-U_{i}\left(1-x_{i}\right) \quad(i=1, \cdots, n) \\
y \leq \sum_{i=1}^{n} \delta_{i}^{(k)} x_{i}^{(k)}(k=1, \cdots, r) \\
\delta_{i}^{(k)} \leq U_{i} w_{i}^{(k)}(k=1, \cdots, r),(i=1, \cdots, n) \\
\delta_{i}^{(k)} \leq \theta_{i}-L_{i}\left(1-w_{i}^{(k)}\right)(k=1, \cdots, r),(i=1, \cdots, n) \\
\sum_{i=1}^{n} w_{i}^{(k)}=1 \quad(k=1, \cdots, r) \\
w_{i}^{(k)} \in\{0,1\}, \quad \delta_{i}^{(k)} \geq 0 \quad(k=1, \cdots, r),(i=1, \cdots, n) \\
y \in \mathbb{R}, z \in \mathbb{R} .
\end{gathered}
$$

\section{Lemma 2.2.}

(i) $F\left(Q L^{(r)}\right) \neq \emptyset$.

(ii) There exists an optimal solution for $Q L^{(r)}$.

(iii) If $\left(\theta^{*}, x^{*}, y^{*}, z^{*}, \delta^{(1)^{*}}, \cdots, \delta^{(r)^{*}}, w^{(1)^{*}}, \cdots, w^{(r)^{*}}\right)$ is an optimal solution for $Q L^{(r)}$ then $\left(\theta^{*}, x^{*}\right)$ is an optimal solution for $Q^{(r)}$ and $v\left(Q^{(r)}\right)=$ $v\left(Q L^{(r)}\right)=y^{*}-z^{*}$. 
Proof. (i) Let $\theta \in \Omega$ and $x \in X$. Let $z=\max \left\{\theta_{1} x_{1}, \cdots, \theta_{n} x_{n}\right\}$. By construction we have:

$$
\begin{aligned}
& z \geq L_{i} x_{i} \quad(i=1, \cdots, n) \\
& z \geq \theta_{i}-U_{i}\left(1-x_{i}\right)(i=1, \cdots, n) .
\end{aligned}
$$

Let $y=\min \left\{\max \left\{\theta_{1} x_{1}^{(k)}, \cdots, \theta_{n} x_{n}^{(k)}\right\}: k=1, \cdots, r\right\}$,

let $i_{k}$ such that $\max \left\{\theta_{1} x_{1}^{(k)}, \cdots, \theta_{n} x_{n}^{(k)}\right\}=\theta_{i_{k}} x_{i_{k}}{ }^{(k)}$,

let $w_{i}^{(k)}=1$ if and only if $i=i_{k}(k=1, \cdots, r),(i=1, \cdots, n)$ and let $\delta_{i}^{(k)}=$ $\theta_{i} w_{i}^{(k)}(k=1, \cdots, r),(i=1, \cdots, n)$. By construction we have:

$$
\begin{gathered}
\delta_{i}^{(k)} \leq U_{i} w_{i}^{(k)}(k=1, \cdots, r),(i=1, \cdots, n) \\
\delta_{i}^{(k)} \leq \theta_{i}-L_{i}\left(1-w_{i}^{(k)}\right)(k=1, \cdots, r),(i=1, \cdots, n) \\
\sum_{i=1}^{n} w_{i}^{(k)}=1 \quad(k=1, \cdots, r) \\
w_{i}^{(k)} \in\{0,1\}, \quad \delta_{i}^{(k)} \geq 0(k=1, \cdots, r),(i=1, \cdots, n) \\
y \in \mathbb{R}, z \in \mathbb{R} .
\end{gathered}
$$

Finally, we have:

$$
\begin{aligned}
\sum_{i=1}^{n} \delta_{i}^{(k)} x_{i}^{(k)} & =\theta_{i_{k}} x_{i_{k}}^{k}=\max \left\{\theta_{1} x_{1}^{(k)}, \cdots, \theta_{n} x_{n}^{(k)}\right\} \\
& \geq \min \left\{\max \left\{\theta_{1} x_{1}^{(k)}, \cdots, \theta_{n} x_{n}^{(k)}\right\}: k=1, \cdots, r\right\}=y .
\end{aligned}
$$

(ii) $Q L^{r}$ may be viewed as a finite set of linear programming problems. A member of the set is the problem with $x, w^{1}, \cdots, w^{r}$ fixed. By construction of $Q L^{r}$ we have that $y-z$ is bounded and then each linear programming problem has an optimal solution. Therefore $Q L^{r}$ has an optimal solution.

(iii) Let $\left(\theta^{*}, x^{*}, y^{*}, z^{*}, \delta^{(1)^{*}}, \cdots, \delta^{(r)^{*}}, w^{(1)^{*}}, \cdots, w^{(r)^{*}}\right)$ be an optimal solution of $Q L^{r}$. We have that $z^{*} \geq L_{i} x_{i}^{*}$ and $z^{*} \geq \theta_{i}^{*}-U_{i}\left(1-x_{i}^{*}\right)$ for all $i=1, \cdots, n$. Therefore $z^{*} \geq \theta_{i}^{*} x_{i}^{*}$ for all $i=1, \cdots, r$. Since maximization is the optimization criterion then $z^{*}=\max \left\{\theta_{1}^{*} x_{1}^{*}, \cdots, \theta_{n}^{*} x_{n}^{*}\right\}=\phi\left(\theta^{*}, x^{*}\right)$.

We have that

$$
\begin{aligned}
& \delta_{i}^{(k)^{*}} \leq U_{i} w_{i}^{(k)^{*}}(k=1, \cdots, r),(i=1, \cdots, n) \\
& \delta_{i}^{(k)^{*}} \leq \theta_{i}^{*}-L_{i}\left(1-w_{i}^{(k)^{*}}\right) \quad(k=1, \cdots, r),(i=1, \cdots, n)
\end{aligned}
$$

and then $\delta_{i}^{(k)^{*}} \leq \theta_{i}^{*} w_{i}^{(k)^{*}}$ for all $i=1, \cdots, n$ and for all $k=1, \cdots, r$. 
Since $\sum_{i=1}^{n} w_{i}^{(k)^{*}}=1$ and $y^{*} \leq \sum_{i=1}^{n} \delta_{i}^{(k)^{*}} x_{i}^{(k)} \leq \sum_{i=1}^{n} \theta_{i}^{*} w_{i}^{(k)^{*}} x_{i}^{(k)}$ it follows that $y^{*} \leq \max \left\{\theta_{1}^{*} x_{1}^{(k)}, \cdots, \theta_{n}^{*} x_{n}^{(k)}\right\}$ for all $k=1, \cdots, r$. Since maximization is the optimization criterion then $y^{*}=\min \left\{\max \left\{\theta_{1}^{*} x_{1}^{(k)}, \cdots, \theta_{n}^{*} x_{n}^{(k)}\right\}, \quad k=1, \cdots, r\right\}=$ $\min \left\{\phi\left(\theta^{*}, x^{(1)}\right), \cdots, \phi\left(\theta^{*}, x^{(r)}\right)\right\}=g^{(r)}\left(\theta^{*}\right)$. It follows that $Q L^{(r)}$ may be rewritten as

$$
\left(Q^{(r)}\right) \max g^{(r)}(\theta)-\phi(\theta, x) \text { s.t. } \theta \in \Omega, \quad x \in X
$$

and $v\left(Q^{(r)}\right)=v\left(Q L^{(r)}\right)=y^{*}-z^{*}$ with $\left(\theta^{*}, x^{*}\right)$ an optimal solution.

\section{Computational experience}

Previous computational experience in the min sum case was presented in [3]. The problem considered was the multiconstrained 0-1-Knapsack problem. In that case the algorithm was implemented in XL-FORTRAN by using the OSL package of IBM [14] that uses a Branch and Bound algorithm based on linear relaxations to solve MILP problems.

Now our algorithm for the min max case has been implemented in $\mathrm{C}++$ by using the new OSL package of IBM [15]. The new experiments were performed on a PC Pentium IV with 2Ghz and 256 MB of RAM.

The min max problem considered was the bottleneck generalized assignment (BGA) problem (Martello and Toth [13]). Our experimental results are preliminary since more problems should be solved before concluding on certain topics.

We follow exactly the paper of Martello and Toth [13] for the formulation of the BGA problem:

Given $n$ items and $m$ units, the penality, $\theta_{i j}$, and the resource requirement, $r_{i j}$, corresponding to the assignment of item $j$ to unit $i(j=1, \cdots, n ; i=1, \cdots, m)$, and the amount of resource, $a_{i}$, available at unit $i(i=1, \cdots, m)$, the BGA problem is to assign each item to one unit so that the total resource requirement for any unit does not exceed its availability and the maximum penality incurred is minimized. By introducing binary variables $x_{i j}$ with $x_{i j}=1$ if and only if item $j$ is assigned to unit $i$, the problem can be formulated as

$$
\begin{gathered}
\min z=\max \left\{\theta_{i j} x_{i j}: i=1, \cdots, m, j=1, \cdots, n\right\} ; s . t . \\
\sum_{j=1}^{n} r_{i j} x_{i j} \leq a_{i}, \quad i=1, \cdots, m \\
\sum_{i=1}^{m} x_{i j}=1, \quad j=1, \cdots, n \\
x_{i j} \in\{0,1\}, \quad i=1, \cdots m, j=1, \cdots, n .
\end{gathered}
$$

The data were generated using procedures analogous to those used by Martello and Toth, as follows: 
Case 1. The elements $r_{i j}$ were drawn from a uniform distribution on [1,rmax] with $r \max >1$, the elements $a_{i}$ were determined by summing the elements $r_{i j}$ and multiplying this sum by $\alpha(0<\alpha<1)$. The final $r_{i j}$ and $a_{i}$ were obtained by rounding down the generated data $(i=1, \cdots m, j=1, \cdots, n)$. Let $J \subseteq$ $\{1, \cdots, m\} \times\{1, \cdots, n\}$ be the index set of elements of the objective function that will be perturbed. Let $k$ be the cardinality of $J$. The indexes that belong to $J$ were selected at random. If $(i, j) \notin J$ then we use $\theta_{i j}=L_{i j}=U_{i j}$ with $L_{i j}=U_{i j}$ drawn from a uniform distribution on $[1, u \max ]$ with $u \max >1$. If $(i, j) \in J$ then we use $L_{i j} \leq \theta_{i j} \leq U_{i j}$ with $L_{i j}=(1-\beta) z_{i j}$ and $U_{i j}=(1+\beta) z_{i j}$ and $z_{i j}$ drawn from a uniform distribution on [1,umax] and $0<\beta<1$. The final $L_{i j}$ and $U_{i j}$ were obtained by rounding down the generated data $(i=1, \cdots m, j=1, \cdots, n)$.

Case 2. The elements $r_{i j}$ were drawn from a uniform distribution on [1,rmax] with $r \max >1$, the elements $a_{i}$ were determined by summing the elements $r_{i j}$ and multiplying this sum by $\alpha=1 / \mathrm{m}$. The final $r_{i j}$ and $a_{i}$ were obtained by rounding down the generated data $(i=1, \cdots, m, j=1, \cdots, n)$. Let $J \subseteq\{1, \cdots, m\} \times$ $\{1, \cdots, n\}$ be the index set of elements of the objective function that will be perturbed. Let $k$ be the cardinality of $J$. The indexes that belong to $J$ were selected at random. If $(i, j) \notin J$ then we use $\theta_{i j}=L_{i j}=U_{i j}$ with $L_{i j}=U_{i j}$ drawn from a uniform distribution on $\left[1,1+u \max -r_{i j}\right]$ with $u \max \geq r \max$. If $(i, j) \in J$ then we use $L_{i j} \leq \theta_{i j} \leq U_{i j}$ with $L_{i j}=(1-\beta) z_{i j}$ and $U_{i j}=(1+\beta) z_{i j}$ and $z_{i j}$ drawn from a uniform distribution on $\left[1, u \max -r_{i j}\right]$ and $0<\beta<1$. The final $L_{i j}$ and $U_{i j}$ were obtained by rounding down the generated data $(i=1, \cdots, m, j=1, \cdots, n)$.

The experiments were designed in order to evaluate the performance of the algorithm as $m, n, k, \alpha$ or $\beta$ vary. The results are reported in Tables 1 (case 1) and 2 (case 2). The notation used in the tables is as follows: $p$ is an index to identify the problem, $r$ is the number of $Q L$-problems solved in order to complete the multiparametrical analysis, Si1 the number of simplex iterations computed to solve $P\left(\theta^{1}\right)$ (we use $\theta^{1}=L$ in all the experiments), $N 1$ the number of nodes generated by the branch and bound algorithm to solve $P\left(\theta^{1}\right), S i$ the number of simplex iterations computed to solve $P\left(\theta^{1}\right), Q L^{(1)}, \cdots, Q L^{(r)}, N$ the number of nodes generated by the branch and bound algorithm to solve $P\left(\theta^{1}\right), Q L^{(1)}, \cdots, Q L^{(r)}$ and $t$ the CPU time in seconds to solve $P\left(\theta^{1}\right), Q L^{(1)}, \cdots, Q L^{(r)}$. Both, Si1 and Si, include the number of simplex iterations computed to solve the relaxations of subproblems in the branch and bound algorithm.

\section{Summary AND FURTher EXTENSIONS}

We designed and implemented an algorithm to solve the multiparametric min max 0-1-IP problem relative to the objective function. Computational experience was presented for BGA problems with uncorrelated (case 1) and correlated (case 2) data. Our algorithm works by choosing an appropriate finite sequence of non-parametric MILP problems in order to obtain a complete multiparametrical analysis and this explains that the computer storage was not a problem for our algorithm, that is: if we can solve the non-parametric $P\left(\theta^{(1)}\right)$ problem then we 
TABLE 1. Computational results for problems generated according to case 1 .

\begin{tabular}{|c|c|c|c|c|c|c|c|c|c|c|c|c|c|}
\hline$p$ & $m$ & $n$ & $k$ & $u \max$ & $r \max$ & $\alpha$ & $\beta$ & $r$ & Si1 & $N 1$ & $S i$ & $N$ & $\bar{t}$ \\
\hline 1 & 2 & 10 & 10 & 1000 & 1000 & 0.50 & 0.20 & 6 & 40 & 12 & 2129 & 707 & 1.62 \\
\hline 2 & & 25 & & & & & & 7 & 123 & 50 & 23595 & 6236 & 34.18 \\
\hline 3 & & 50 & & & & & & 2 & 236 & 109 & 1062 & 273 & 0.73 \\
\hline 4 & & 100 & & & & & & 2 & 312 & 104 & 1901 & 460 & 3.01 \\
\hline 5 & & 150 & & & & & & 1 & 696 & 169 & 1891 & 536 & 43.75 \\
\hline 6 & 3 & 10 & 10 & 1000 & 1000 & 0.50 & 0.20 & 7 & 73 & 24 & 7937 & 2072 & 6.96 \\
\hline 7 & & 25 & & & & & & 7 & 1556 & 573 & 6174 & 1331 & 36.71 \\
\hline 8 & & 50 & & & & & & 4 & 581 & 177 & 7081 & 1422 & 48.26 \\
\hline 9 & & 100 & & & & & & 2 & 3023 & 1848 & 61303 & 34938 & 360.04 \\
\hline 10 & 5 & 10 & 10 & 1000 & 1000 & 0.50 & 0.20 & 6 & 172 & 60 & 4837 & 1182 & 4.48 \\
\hline 11 & & 25 & & & & & & 4 & 2883 & 1053 & 4869 & 1591 & 4.77 \\
\hline 12 & & 50 & & & & & & 1 & 13197 & 3692 & 13856 & 3884 & 9.00 \\
\hline 13 & 10 & 10 & 10 & 1000 & 1000 & 0.50 & 0.20 & 2 & 3638 & 755 & 4354 & 1015 & 1.62 \\
\hline 14 & & 25 & & & & & & 2 & 14057 & 2877 & 15594 & 3431 & 10.05 \\
\hline 15 & 2 & 10 & 10 & 1000 & 1000 & 0.50 & 0.05 & 4 & 27 & 3 & 639 & 80 & 0.16 \\
\hline 16 & & & & & & & 0.10 & 5 & 49 & 18 & 920 & 166 & 0.28 \\
\hline 17 & & & & & & & 0.15 & 7 & 43 & 12 & 15622 & 3124 & 8.76 \\
\hline 18 & & & & & & & 0.20 & 7 & 38 & 10 & 12807 & 3285 & 8.34 \\
\hline 19 & & & & & & & 0.25 & 14 & 22 & 54 & 55101 & 14101 & 71.72 \\
\hline 20 & & & & & & & 0.30 & 15 & 118 & 34 & 54452 & 12183 & 186.42 \\
\hline 21 & 2 & 120 & 10 & 100 & 100 & 0.50 & 0.20 & 2 & 17513 & 11112 & 20158 & 11841 & 32.94 \\
\hline 22 & & 140 & 8 & & & & & 1 & 61996 & 38116 & 62826 & 38260 & 98.54 \\
\hline 23 & & 160 & 6 & & & & & 2 & 14037 & 9701 & 38342 & 13436 & 2112.5 \\
\hline 24 & 2 & 10 & 10 & 1000 & 1000 & 0.35 & 0.20 & 2 & 80 & 32 & 427 & 154 & 0.13 \\
\hline 25 & & & & & & 0.40 & & 5 & 59 & 15 & 1628 & 434 & 0.73 \\
\hline 26 & & & & & & 0.45 & & 3 & 111 & 50 & 1182 & 382 & 0.60 \\
\hline 27 & & & & & & 0.50 & & 3 & 56 & 16 & 619 & 192 & 0.23 \\
\hline 28 & & & & & & 0.55 & & 10 & 61 & 33 & 118338 & 21285 & 89.44 \\
\hline 29 & & & & & & 0.60 & & 9 & 116 & 63 & 11389 & 2460 & 8.65 \\
\hline 30 & & & & & & 0.65 & & 12 & 43 & 15 & 14232 & 2238 & 9.31 \\
\hline 31 & 2 & 10 & 10 & 10 & 1000 & 0.50 & 0.20 & 3 & 27 & 4 & 540 & 70 & 0.13 \\
\hline 32 & & & & 100 & & & & 5 & 85 & 33 & 2313 & 583 & 1.17 \\
\hline 33 & & & & 10000 & & & & 11 & 88 & 13 & 15911 & 3805 & 15.66 \\
\hline 34 & $\overline{2}$ & 10 & 10 & 1000 & 10 & 0.50 & 0.20 & 5 & 59 & 23 & 1563 & 306 & 0.68 \\
\hline 35 & & & & & 100 & & & 3 & 29 & 14 & 412 & 78 & 0.11 \\
\hline 36 & & & & & 10000 & & & 5 & 54 & 17 & 1565 & 352 & 0.67 \\
\hline 37 & 2 & 25 & 15 & 1000 & 1000 & 0.50 & 0.20 & 3 & 101 & 34 & 1033 & 257 & 0.46 \\
\hline 38 & & & 20 & & & & & 7 & 8043 & 5096 & 84933 & 21792 & 102.06 \\
\hline 39 & & & 25 & & & & & 7 & 1295 & 738 & 37723 & 11044 & 54.45 \\
\hline
\end{tabular}

can expect no problems to perform a complete multiparametrical analysis. The algorithm may be implemented by using any software capable of solving MILP problems. To the best of our knowledge there are no other implementations of algorithms to solve the multiparametric min max 0-1-IP problem relative to the objective function and for this reason we did not compare the performance of our algorithm with any other.

A generalization of $Q L^{(r)}$ may be carefully designed by using analogous linearization techniques used in the min sum and min max cases in such a manner that the following case may be considered:

$$
\phi(\theta, x)=\max \left\{\left(F^{(1)} \theta\right)^{t} x+d^{(1)^{t}} \theta+c^{(1)^{t}} x, \cdots,\left(F^{(k)} \theta\right)^{t} x+d^{(k)^{t}} \theta+c^{(k)^{t}} x\right\}
$$

where $F^{(i)} \in \mathbb{R}^{n \times p}, d^{(i)} \in \mathbb{R}^{p}, c^{(i)} \in \mathbb{R}^{n}(i=1, \cdots, k)$.

The min sum and min max are particular cases of this general case.

In non-parametric 0-1-IP a significant effort is directed towards the design of special purpose algorithms for problems with particular structures. It is reasonable then to think that a next step should be the design of specialized multiparametric algorithms in order to solve hard problems with higher dimensions. The multiparametric algorithm turns out to be, from this point of view, a general methodology and problems $Q L^{(r)}$ would be solved with specialized algorithms associated to the structure of the problems $P(\theta)$. 
TABLE 2. Computational results for problems generated according to case 2 .

\begin{tabular}{|c|c|c|c|c|c|c|c|c|c|c|c|c|c|}
\hline$p$ & $m$ & $n$ & $k$ & $u \max$ & $r \max$ & $\alpha$ & $\beta$ & $r$ & Si1 & $N 1$ & $\overline{S i}$ & $N$ & $t$ \\
\hline 1 & 2 & 10 & 10 & 1000 & 1000 & 0.50 & 0.20 & 7 & 35 & 11 & 8548 & 2071 & 5.13 \\
\hline 2 & & 25 & & & & & & 6 & 151 & 50 & 6226 & 1921 & 9.35 \\
\hline 3 & & 50 & & & & & & 2 & 603 & 304 & 3883 & 2126 & 8.42 \\
\hline 4 & & 100 & & & & & & 2 & 748 & 243 & 2463 & 793 & 4.64 \\
\hline 5 & & 150 & & & & & & 1 & 1560 & 680 & 446223 & 42522 & 256.43 \\
\hline 6 & 3 & 10 & 10 & 1000 & 1000 & 0.33 & 0.20 & 3 & 603 & 214 & 1595 & 497 & 0.52 \\
\hline 7 & & 25 & & & & & & 2 & 25050 & 5138 & 27062 & 5807 & 7.76 \\
\hline 8 & & 50 & & & & & & 2 & 113451 & 10154 & 557349 & 32885 & 1102.8 \\
\hline 9 & & 100 & & & & & & 1 & 310443 & 43231 & 640223 & 145845 & 1980.1 \\
\hline 10 & 5 & 10 & 10 & 1000 & 1000 & 0.20 & 0.20 & $\frac{2}{2}$ & 4085 & 741 & 5802 & 1064 & 1.21 \\
\hline 11 & & 25 & & & & & & 3 & 112527 & 9356 & 673418 & 134873 & 1167.2 \\
\hline 12 & & 50 & & & & & & 2 & 215167 & 32111 & 573672 & 112317 & 1423.9 \\
\hline 13 & 10 & 10 & 10 & 1000 & 1000 & 0.10 & 0.20 & 1 & 205449 & 52621 & 619509 & 164972 & 319.37 \\
\hline 14 & & 25 & & & & & & 1 & 342156 & 39147 & 549669 & 136239 & 1004.2 \\
\hline 15 & 2 & 10 & 10 & 1000 & 1000 & 0.50 & 0.05 & 2 & 68 & 28 & 298 & 91 & 0.05 \\
\hline 16 & & & & & & & 0.10 & 2 & 72 & 36 & 311 & 123 & 0.09 \\
\hline 17 & & & & & & & 0.15 & 3 & 80 & 33 & 1049 & 256 & 0.29 \\
\hline 18 & & & & & & & 0.20 & 1 & 50 & 14 & 125 & 27 & 0.03 \\
\hline 19 & & & & & & & 0.25 & 2 & 54 & $\begin{array}{l}22 \\
22\end{array}$ & 441 & 160 & $\begin{array}{l}0.12 \\
0.12\end{array}$ \\
\hline 20 & & & & & & & 0.30 & 5 & 120 & 44 & $\begin{array}{r}7109 \\
\end{array}$ & 2815 & 5.38 \\
\hline 21 & 2 & 120 & 10 & 100 & 100 & 0.50 & 0.20 & 2 & 12923 & 6142 & 18222 & 9755 & 35.43 \\
\hline 22 & & 140 & 8 & & & & & 2 & 24521 & 12281 & 45168 & 21311 & 136.22 \\
\hline 23 & & 160 & 6 & & & & & 2 & 13182 & 6112 & 28513 & 14623 & 60.41 \\
\hline 24 & 2 & 10 & 10 & 1000 & 1000 & 0.35 & 0.20 & 4 & 46 & 13 & 1285 & 311 & 0.45 \\
\hline 25 & & & & & & 0.40 & & 1 & 55 & 19 & 147 & 36 & 0.02 \\
\hline 26 & & & & & & 0.45 & & 2 & 57 & 31 & 341 & 68 & 0.04 \\
\hline 27 & & & & & & 0.50 & & 4 & 155 & 94 & 933 & 259 & 0.26 \\
\hline 28 & & & & & & 0.55 & & 2 & 112 & 62 & 390 & 144 & 0.11 \\
\hline 29 & & & & & & 0.60 & & 5 & 42 & 20 & 1378 & 345 & 0.59 \\
\hline 30 & & & & & & 0.65 & & 5 & 41 & 16 & 3147 & 683 & 1.38 \\
\hline 31 & $\overline{2}$ & 25 & 15 & 1000 & 1000 & 0.50 & 0.20 & $\overline{2}$ & 65348 & 41351 & 65953 & 41551 & 525.34 \\
\hline 32 & & & 20 & & & & & 5 & 152 & 50 & 19648 & 7613 & 30.31 \\
\hline 33 & & & 25 & & & & & 5 & 171 & 80 & 6507 & 2829 & 10.79 \\
\hline
\end{tabular}

Recently we have presented (see Crema [5]) a unified approach, that can be viewed as a generalization of [2-4], to perform a complete multiparametrical analysis to 0-1-ILP problems with the perturbation of the right-hand-side vector, the objective function and the constraint matrix simultaneously considered. In a near future we will intend to develop a unified approach to solve, if possible, the general min max multiparametric 0-1-IP problem with all the perturbations simultaneously considered.

\section{REFERENCES}

[1] H. Arsham, http://ubmail.ubalt.edu/ harsham/refop/Refop.htm

[2] A. Crema, A contraction algorithm for the multiparametric integer linear programming problem. Eur. J. Oper. Res. 101 (1997) 130-139.

[3] A. Crema, An algorithm for the multiparametric 0-1-integer linear programming problem relative to the objective function. Eur. J. Oper. Res. 125 (2000) 18-24.

[4] A. Crema, An algorithm for the multiparametric 0-1-integer linear programming problem relative to the constraint matrix. Oper. Res. Lett. 27 (2000) 1-46.

[5] A. Crema, The multiparametric 0-1-Integer Linear Programming problem: A unified approach. Eur. J. Oper. Res. 139 (2002) 511-520.

[6] A.M. Geoffrion and R. Nauss, Parametric and postoptimality analysis in integer linear programming. Manage. Sci. 23 (1977) 453-466. 
[7] H.J. Greenberg, An annoted bibliography for post-solution analysis in mixed integer propgramming and combinatorial optimization, Advances in Computational and Stochastic Optimization, Logic Programming and Heuristic Search, edited by D.L. Woodruff. Kluwer Academic Publishers, Boston, MA (1998) 97-148.

[8] H.J. Greenberg, An annoted bibliography for post-solution analysis in mixed integer programming and combinatorial optimization, http://carbon.cudenver.edu/ hgreenbe/aboutme/pubrec.html

[9] L. Jenkins, Parametric methods in integer linear programming. Ann. Oper. Res. 27 (1990) $77-96$.

[10] L. Jenkins, Parametric mixed integer programming: an application to solid waste management. Manage. Sci. 28 (1982) 1270-1284.

[11] L. Jenkins, Using parametric integer programming to plan the mix of an air transport fleet. INFOR 25 (1987) 117-135.

[12] L. Jenkins, Parametric-objective integer programming using knapsack facets and Gomory cutting planes. Eur. J. Oper. Res. 31 (1987) 102-109.

[13] S. Martello and P. Toth, The bottleneck generalized assignment problem. Eur. J. Oper. Res. 83 (1995) 621-638.

[14] Optimization Subroutine Library, release 2, Guide and Reference, IBM (1992).

[15] IBM Optimization Library C, Application Programming Interface. Available at http://www-306.ibm.com/sof tware/data/bi/osl/pubs/library/featCAPI.htm

[16] M. Oral and O. Kettani, A linearization procedure for quadratic and cubic mixed integer problems. Oper. Res. 40 (1992) S109-S116.

[17] B. Thiongane, A. Nagih and G. Plateau, Theoretical and algorithmic study for parametric 0-1 linear programs relative to the objective function. Math. Program, submitted. 